\title{
SIMPLE QUALITATIVE DETERMINISTIC MODEL OF DYNEIN
}

\author{
J. Rosenberg*, M. Krejčová**
}

\begin{abstract}
The paper is devoted to the development of a simple model of dynein. Such a model should allow for the study of the influence of different parts of dynein on its motion. The importance of this model is connected with the growing knowledge of the influence of dynein on some serious neurodegenerative diseases. The introduced model is based on the simulation of the Brownian motion using time reversible thermostated oscillators. Model allows to model also such effect as the catch-bond.
\end{abstract}

Keywords: molecular motors, processivity, dynein, Brownian motion, catch bond effect

\section{Introduction}

One of the most important processive molecular motors seems to be dynein. According to current knowledge, dynein is important because of its connection to neurodegenerative diseases like Parkinson, Alzheimer and ALS (Chen et al. (2014)). It plays an important role in glaucoma too (Calkins (2008)). So far, there is a whole family of dynein motors that are well known. They can have a different number of heads. The cytoplasmic dynein (Fig. 1) has two heads and we will focus on this type here.

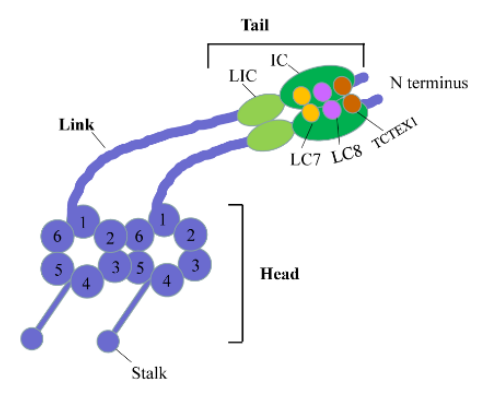

Fig. 1: Scheme of cytoplasm dynein. Two heads are connected with a single tail. Adapted from Carter (2013)

Its structure and motion are different in comparison with other processive molecular motors, like kinesin or myosin V. The main parts of dynein and their names are shown in Fig. 2a (Roberts et al. (2013)).

The whole mechanochemical cycle is described e.g. in Belyy et al. (2014) as following:

The head (motor domain) consists of six subunits AAA+ ATPase arranged into a hexameric ring (Fig. 2a). Four of the AAA+ subunits bind nucleotide and the AAA1 subunit serves as the primary site of ATP. When an ATP molecule binds to the AAA1 site the head is released from the binding site on the microtubule (MT) (Fig. 2b).

The linker concurrently crosses the AAA+ ring doing the priming stroke. This stroke moves the stalk and microtubule binding domain (MTBD) towards the negative end of MT. After hydrolysis of ATP the

Prof. Ing. Josef Rosenberg, DrSc.: Department of Mechanics, University of West Bohemia, Univerzitní 22; 314 06, Pilsen; CZ, rosen@kme.zcu.cz

** Ing. Milada Krejčová: Department of Mechanics, University of West Bohemia, Univerzitní 22; 314 06, Pilsen; CZ, mkrejcov@ntis.zcu.cz 
head again binds to MT at a new position. The inorganic phosphate is released, and the linker is returned to the starting unprimed state - it does the power stroke. The head is moving ahead. In this phase the mechanical work is produced.
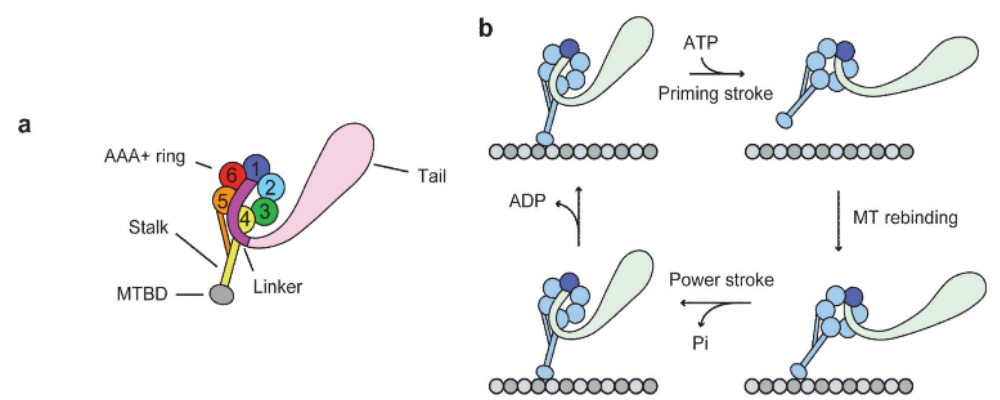

Fig. 2: a) Detailed scheme of dynein; b) mechanochemical dynein cycle. Adapted from Roberts et al. (2013); Belyy et al. (2014).

This was the description of the mechanochemical cycle of one motor domain. As was mentioned above, the whole cytoplasmic dynein motor consists of two of these domains and can perform a continuous motion along the microtubule. One stalk is always bound to the MT, so this motor is called the processive one. During its motion, it can bring the cargo from the + end of MT (e. g. membrane) to the - end (e. g. nucleus). The cargos can be different organelles or even viruses. It should also that be noted, dynein exhibits s. c. catch bond effect (Nair et al. (2016)) - with the increasing force the unbinding rate decreases. This happens only in a certain range of force.

There exist some computer models of dynein. Let us mention, e.g. pure deterministic model (Crossley et al. (2012)), stochastic model (Goedecke and Elston (2005)) or the very simple model (Zhao et al. (2014)) which omits e.g. the possibility of the backward stepping. Goedecke's model is very complicated and is based on the Langevin equation for the simulation of the diffusion motion.

Our aim is to develop a relatively simple model which takes into account the complicated structure of dynein, all phases of the steps and the diffusion motion of the stalks and cargo.

\section{Deterministic model of the dynein}

The Brownian motion is modelled by the Nose-Hoover oscillator (NH) (Posch et al. (1986)) during the phase when the stalk is detached from the MT caused by ATP, see Fig. 3. After hydrolysis of ATP the potential $W$ causes strong binding of the MTBD with the MT. This potential (or the free energy) generally quantifies the interactions of the moving object (MTBD) with the isothermal environment (here MT; Ruizheng (2013)). The place of the binding position is in the minimum of the potential of $W$. The choice of the minimum depends on the MTBD current position - the last position of the Nose-Hoover oscillator. If the size of the attractor is large enough, the stalk can do one, two or even more steps with the length $L$ forward or backwards.

The potential $W$ has the usual form

$$
W=U_{0}\left(\sin \frac{2 \pi}{L} x+\frac{1}{4} \sin \frac{4 \pi}{L} x\right),
$$

where $U_{0}$ is a characteristic parameter of MT and $L$ is the period of periodical and unsymmetrical potential. Because we only need the gradient of this potential, we can omit the additive constant.

In the Fig. 3, the centre of the attractor of NH is in the point of the potential minimum (last attachment point). To respect the power stroke caused by the priming of the stalk, the centre of the attractor is moved on the value $p s$. Under the action of the external force Fload, the attached stalk is deformed in the direction of this force on the amount Fload - invstiff. The deformation also causes the movement of the centre of the attractor. In the Fig. 4, there is a timeline with the periods of the Brownian motion and the periods when the MTBD is attached to the MT. Here $f f$ is the sampling period, $4 /(2 \nu)$ is the minimal step length and $k k$ corresponds with the detachment rate. 


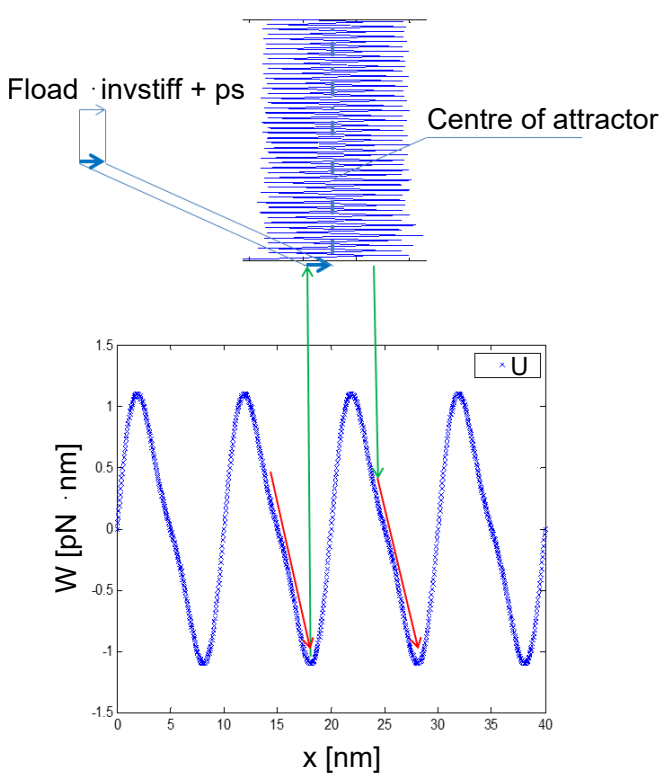

Fig. 3: Nose-Hoover oscillator for the Brownian motion simulation. $\mathrm{cb} 4 \cdot 1 /(2 \mathrm{v}) \cdot \mathrm{cb} \quad \Delta \mathrm{cb} 4 \cdot 1 /(2 \mathrm{v}) \cdot \mathrm{cb}+\Delta \mathrm{cb}$

$1 /(2 v)$

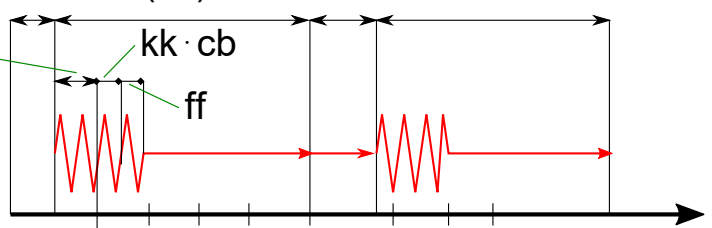

To model the catch bond effect, the time period, the parameter $\nu$ and the parameters $k k$ were changed and parameters $c b$ and $\Delta c b$ were introduced.

According to the above-mention algorithm two codes in MATLAB were developed - Dynein $4 . \mathrm{m}$ and Dynein $6 . m$. The last one contents the catch bond effect.

\section{Results and discussion}

At first, we present the case with Fload $=0 \mathrm{pN}$. The results obtained with Dynein $4 . \mathrm{m}$ are shown in Fig. 5. All the possible steps are visible there - see the circles. It should be stressed that these results are only qualitative. Further development is necessary to obtain the physiological values.
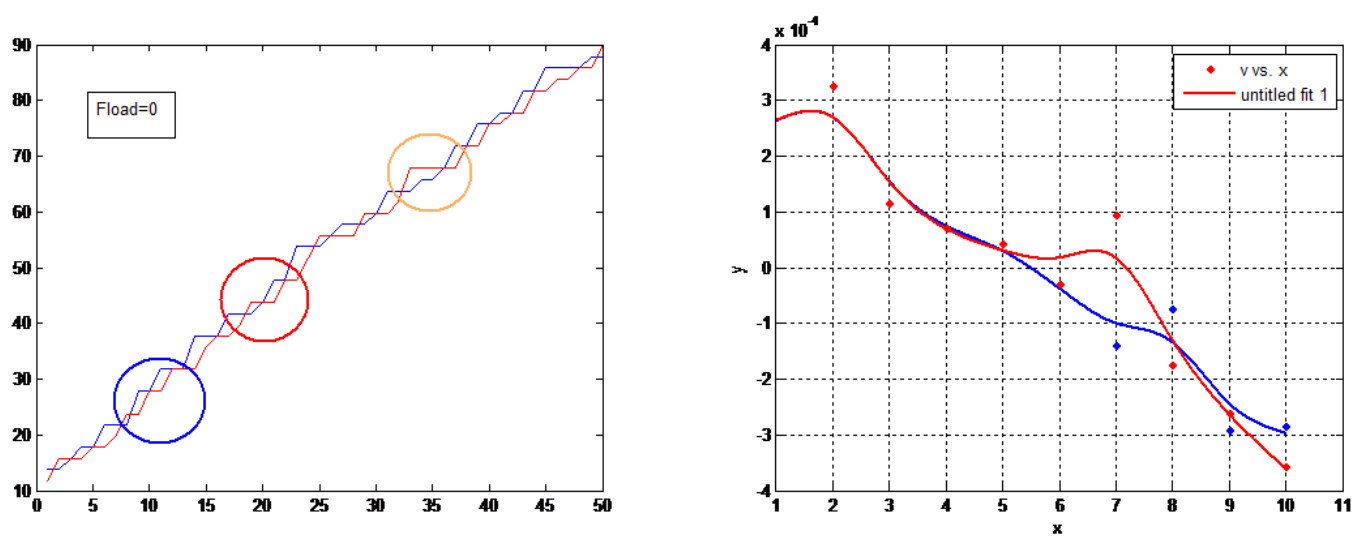

Fig. 5: Length of motion (vertical) in the particular steps: red circle - "hand-over-hand", blue circle - "inchworm", orange circle represents not alternating nor passing steps. Different lines colours correspond with both stalks. The values Fig. 6: Dependence Load (x) vs. velocity (y). Red line --catch-bond effect, blue line - without catch-bond effect. are only qualitative.

In Fig. 6, we can see the dependence between load and the velocity. The red line corresponds to the catch bond effect starting with Fload $=-6 \mathrm{pN}$. These results can be compared with results in Johnson (2016), see Fig. 7. 


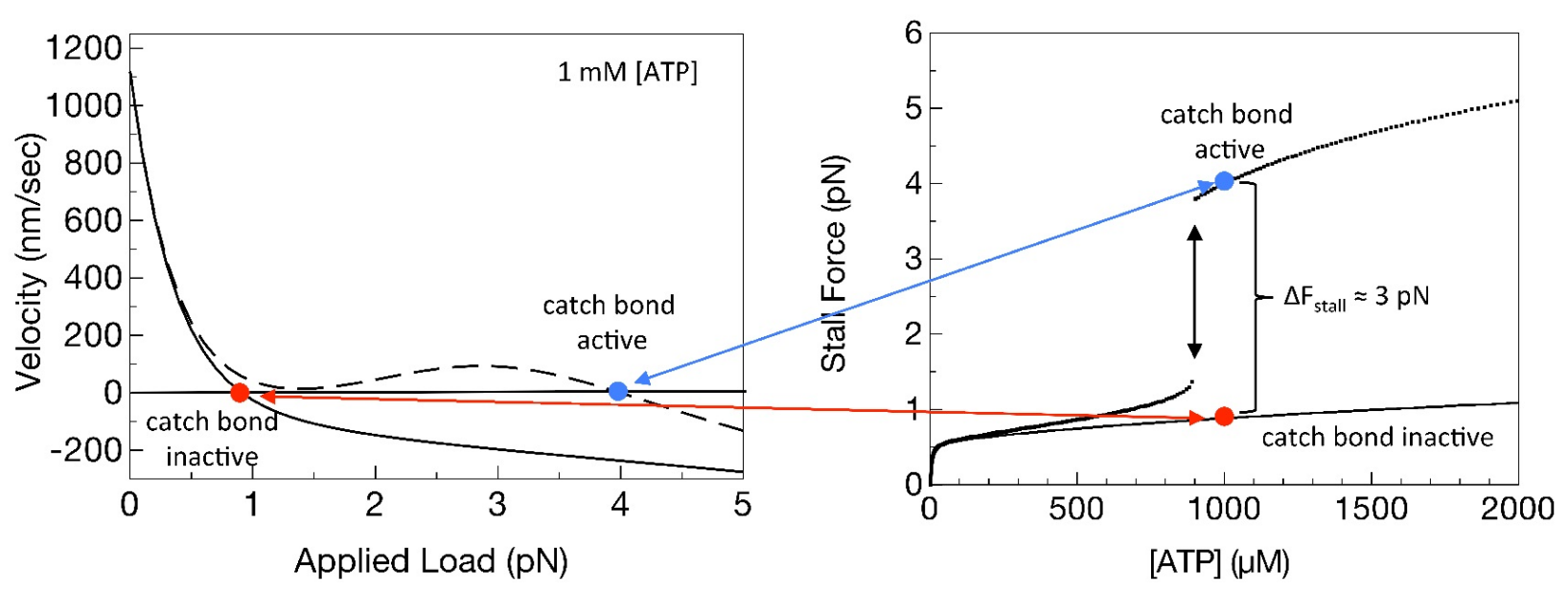

Fig. 7: Experiment result from Johnson (2016).

\section{Conclusions}

According to the above-mentioned algorithm we developed two codes in MATLAB - Dynein $4 . m$ and Dynein $6 . \mathrm{m}$. The last one contents the catch-bond effect. Both codes allow the qualitative analysis of all introduced parameters $\left(U_{0}, L, p s\right.$, invstiff, $\left.c b, \Delta c b, \nu, k k\right)$ and the parameters of the oscillator on the dynein motion. Further research will be focused on the fitting the parameters to a real situation.

\section{Acknowledgments}

Krejčová was supported by the projects LO1506 of the Czech Ministry of Education, Youth and Sports and SGS-2016-038.

\section{References}

Belyy, V., Hendel, N. L., Chien, A., Yildiz, A. (2014), Cytoplasmic Dynein Transports Cargos via Load-Sharing between the Heads. Nat Commun. Vol 5, No. 5544.

Calkins, D. J. (September/October 2008), A neurological perspective on glaucoma. Glaucoma today.

Carter, A. P. (2013), Crystal clear insights into how the dynein motor moves. Journal of Cell Science 126, Vol 126, pp 705-713.

Chen, X. J., Xu H., Cooper H. M., Liu Y. B. (2014), Cytoplasmic dynein: a key player in neurodegenerative and neurodevelopmental diseases. Sci China Life Sci, Vol 57, pp 372-377.

Crossley, L., Garrett, C. A., Hafezparast, M., Madzvamuse, A. (2012), From the Cell Membrane to the Nucleus: Unearthing Transport Mechanisms for Dynein. Bull Math Biol, Vol 74, pp 2032-2061.

Goedecke, D. M, Elston, T. C. (2005), A model for the oscillatory motion of single dynein molecules. Journal of Theoretical Biology, Vol 232, pp 27-39.

Johnson, C. M. (2016), Investigating the Slow Axonal Transport of Neurofilaments: A Precursor for Optimal Neuronal Signaling. Thesis of the College of Arts and Sciences of Ohio University.

Nair, A., Chandel, S., Mitra, M. K., Muhuri, S., Chaudhuri, A. (2016), Catch bond mechanism in Dynein motor driven collective transport. ArXiv e-prints.

Posch, H. A., Hoover, W. G., Vesely, F. J. (1986), Canonical dynamics of the Nose oscillator: Stability, order, and chaos. Physical review A, Vol 33, No. 6, pp 4253-4265.

Roberts, A. J., Kon, T., Knight, P., Sutoh, K., Burgess, S. A. (November 2013) Functions and mechanics of dynein motor proteins. Nat. Rev. Mol. Cell Biol., Vol 14(11), pp 713-726.

Ruizheng, H. (2013), Termodynamics and mechanics of molecular motors. A thesis submitted for degree of philosophy. Department of physics. National university of Singapore, Singapore.

Zhao, X. Y., Sun, W., Zhang J. P., Tala, Guo, W. S. (2014), A model for the coordinated stepping of cytoplasmic dynein. Biochemical and Biophysical Research Communications, Vol 453, Issue 4, pp 686-691. 